\title{
Value of transabdominal bowel ultrasonography and splanchic vascular parameters in the evaluation of inflammatory bowel disease
}

\author{
Manal Kamel $^{1,2}$, Magdy El-Serafy ${ }^{1,2}$, Hussein Attia ${ }^{3}$, Hanan Abdeel-Haleem ${ }^{1,2}$, Naglaa Zayed ${ }^{1,2^{*}}$, \\ Maha Hasab-Allah ${ }^{1,2}$, Zakaria Salama ${ }^{1,2}$ \\ ${ }^{1}$ Endemic Medicine Department, Cairo University, Cairo, Egypt \\ ${ }^{2}$ Gastrointestinal Endoscopy Unit, Faculty of Medicine, Cairo University, Cairo, Egypt \\ ${ }^{3}$ Radiology Department, National Cancer Institute, Cairo University, Cairo, Egypt \\ Email: ${ }^{*}$ naglaazayed@yahoo.com
}

Received 25 January 2013; revised 20 March 2013; accepted 28 April 2013

Copyright (C) 2013 Manal Kamel et al. This is an open access article distributed under the Creative Commons Attribution License, which permits unrestricted use, distribution, and reproduction in any medium, provided the original work is properly cited.

\begin{abstract}
Background: Although non-specific, transabdominal bowel ultrasonography, in real time or in color Doppler; has been beneficial in evaluating the location, extent and activity of inflammatory bowel disease (IBD). Aim: To assess the potential role of bowel transabdominal bowel ultrasonography in the diagnosis and follow-up of IBD. Patients and Methods: A cohort of 30 adults histologically-proven active IBD patients; ulcerative colitis (UC) $(n=20)$, Crohn's disease (CD) $(n=10)$ were prospectively assessed in addition to 20 non-IBD participants representing the control group. All participants underwent the full colonoscopy in addition to real time and color Doppler ultrasonography. Bowel wall thickness, peri-enteric changes, and hemodynamic changes in the portal vein and mesenteric arteries were recorded at initial enrollment and after complete remission in $\mathbf{1 0}$ IBD patients. Results: Bowel wall was significantly thickened in all active IBD patients with a significant decrease after disease quiescence in CD patients; $p$ value $<0.01$. At initial evaluation, hypervascularization was evident in both mesenteric arteries among patients with active pancolitis and $C D$ with significant differences; $p$ value was $<\mathbf{0 . 0 0 1}$ after disease quiescence. The diagnostic accuracy of UC and CD lesions ranged from $70 \%-85 \%, 86 \%-100 \%$ respectively. Conclusion: Transabdominal bowel ultrasonography supplemented with color Doppler study may represent a potential tool in the diagnosis, assessment of disease activity as well as follow-up of IBD.
\end{abstract}

"Corresponding author.
Keywords: Bowel Ultrasonography-Inflammatory Bowel Disease; Ulcerative Colitis; Crohn's Disease; Splanchnic Vessels; Doppler

\section{INTRODUCTION}

The term inflammatory bowel disease (IBD) denotes a genetically, immunologically and histopathologically heterogeneous group of bowel disorders classified as ulcerative colitis (UC), Crohn's disease (CD) and indeterminate colitis (IC) [1]. The diagnosis of IBD is based on a non-strictly defined combination of clinical presentation with confirmatory evidence from radiographic, endoscopic and pathological findings [2]. Characteristically, $\mathrm{CD}$ runs a relapsing course and $\mathrm{UC}$ has frequent exacerbations and remissions [3] which explain why these patients are repeatedly investigated by invasive endoscopic and radiologic procedures. However, ileo-colonoscopy is not always possible due to technical difficulties, poor preparation, or patient intolerance [4]. In contrast, computed tomography, magnetic resonance imaging and ultrasonography [1] represent useful alternative tools for the assessment of bowel wall abnormalities and extraluminal alterations [5].

Trans-abdominal ultrasonography of the bowel is a fast, efficient, low-cost, well accepted and repeatable procedure. Being virtually hazard-free, it is considered an important noninvasive procedure of examining the intestines that need to be performed very accurately and requires adequate trained operators [6]. Moreover, Doppler ultrasonography is a well documented technique to assess the splanchnic blood flow and thus can be a valuable method for the assessment of IBD activity [7]. Despite several attempts to correlate US findings with clinical 
and biochemical activity, to our knowledge, there is no standardized scale or convincing data that can determine the degree of disease activity on US, neither for CD nor for UC. Moreover, the possible incorporation of bowel US in the diagnostic panel for IBD has been of major concern due to the presence of controversy regarding this issue.

The aim of this study is to assess the potential role of transabdominal bowel ultrasonography (real time and color Doppler) in the diagnosis, location, and follow up in patients with known IBD and validate these findings in relation to the colonoscopic findings.

\section{MATERIAL AND METHODS}

This prospective study was conducted on a cohort of patients recruited from the Gastrointestinal Endoscopy Unit, Cairo University, Egypt, from January 2004 to January 2007 when colonoscopy was clinically indicated for diagnosis of suspected IBD $(n=30)$. In addition, 20 symptomatic patients with no evidence of gross pathology on colonoscopy were recruited as the control group. Approval was obtained from the ethical research committee of our institution and informed consent was obtained from all participants. Patients with history of colonic resection, clinical suspicion of toxic megacolon or documented splanchnic vessel occlusion were excluded.

The diagnosis of IBD; pan ulcerative colitis $(\mathrm{n}=10)$, left sided colitis $(n=10)$ or $C D(n=10)$; was established through an assessment of the clinical presentation with confirmatory evidence from radiography, ileo-colonoscopy, in addition to pathological findings. The hallmarks for the endoscopic diagnosis of UC were the presence of diffuse involvement in the form of erythema, erosions, shallow ulcerations, friable mucosa and spontaneous bleeding. Whereas the presence of skip lesions comprising focal, patchy erosions or ulcers, vertical fissures, fistulas and the evidence of terminal ileal affection with rectal sparing were suggestive of $\mathrm{CD}$. The severity of both entities was done on subjective evaluation of the endoscopic picture. Concerning histopathological diagnosis, patients with $\mathrm{CD}$ had transmural inflammation with multiple lymphoid aggregates, granulomas and submucosal fibrosis had an evidence of non-caesting granuloma while patients with UC expressed increased chronic inflammatory cells in the lamina propria, neutrophilic infiltration of the surface and crypt epithelium and crypt abscesses.

Routine laboratory investigations such as complete blood picture, stool analysis, C-reactive protein (CRP), and erythrocyte sedimentation rate (ESR) were performed to assess disease activity as determined by Mayo scoring system [8], as well as Crohn's disease activity index score(CDAI) $[9,10]$ for $\mathrm{UC}$ and $\mathrm{CD}$ respectively.

In accordance with the study protocol, transabdominal bowel ultrasonography; real time and color Doppler; was carried out using a real time US apparatus (Siemens, versa-plus ${ }^{\circledR}$, Germany) and a $3.5 \mathrm{MHz}$ curved array at baseline enrollment and was re-evaluated after disease remission. Follow-up; mean $180 \pm 60$ days; was done in 19 IBD patients. Disease remission, as determined by CDAI score $<150$ and Mayo scorning system, was achieved in 10 patients.

Bowel US was performed within 3 days of the endoscopic procedure by a single experienced operator who was blind of any previous diagnoses or diagnostic procedures. All patients were examined in the supine position with no additional dietary or cleansing measures during preparation except fasting for at least 4 hours prior to examination. The bowel loops were examined for wall thickness, presence of skip lesions, inter-bowel or free peritoneal fluid, abscesses, stenotic lesions and fistula. Bowel wall thickness (BWT) was measured from the serosa to the intestinal mucosa at the thickest bowel loop of each bowel segment; $\geq 4 \mathrm{~mm}$ for at least $4 \mathrm{~cm}$ length; was classified as diseased provided it could be measured in both longitudinal and transverse sections [11]. Sigmoid and ileocecal regions were identified by the left and right iliac vessels [12]. Color Doppler scanning of the thickened bowel loops reported the number of signals in the affected bowel loop and evaluated hemodynamic vascular changes in the portal vein (PV) and both the superior and inferior mesenteric arteries (SMA, IMA). All vessels were assessed for their diameter, velocity and blood flow volume which were recorded at an angle of insonation between $30^{\circ}$ and $60^{\circ}$. The scanned arteries were dynamically explored with a $3.5-4 \mathrm{MHz}$ probe, followed longitudinally for a distance of $3-5 \mathrm{~cm}$ and further evaluated for their peak systolic velocity (PSV), end diastolic velocity (EDV), resistivity index (RI) and pulsatility index (PI). All measurements were made during three consecutive flowmetry spectra waveforms.

Blood flow volume was assessed according to the following equation:

Flow volume $=$ mean velocity $(\mathrm{cm} / \mathrm{second}) \times$ crosssectional area $\left(\mathrm{cm}^{2}\right)$.

Statistical methods: All patients' data were tabulated, analyzed by using SPSS 10.0 for window XP quantitative variables were expressed by mean, standard deviation (STD) and compared by ANOVA test, paired or unpaired, $t$-student test when appropriate, and assuming normal distribution of the data. Receiver operator characteristic (ROC) curves were done for the Doppler parameters to detect the most reliable index for diagnosis and its best cut off value. In all tests $p$ value was considered significant when $\leq 0.05$.

\section{RESULTS}

IBD patients were significantly younger than non-IBD 
patients; mean (SD) age for UC patients was 26.25 (7.28) and 21.70 (3.59) years for CD patients, compared to 34.60 (4.0) years in non-IBD patients. Salient features of UC patients were chronic bloody diarrhea, tenesmus, and localized left iliac fossa tenderness, stool analysis positive for blood and pus, and anemia as shown in Table 1. In $\mathrm{CD}$ patients, diarrhea, abdominal cramps, tenderness of the right iliac fossa, weight loss and perianal disease were most frequent.

The colonoscopic and ultrasonographic findings in all IBD were described in Table 2. Colonoscopy revealed the picture of either pan-colitis $(50 \%)$ or left-sided colitis $(50 \%)$ with no right colon involvement in UC while transabdominal bowel US was able to demonstrate an overall $70 \%$ diagnostic accuracy; $70 \%$ and $85 \%$ in pan and left-sided colitis respectively. In $\mathrm{CD}$, colonoscopy was able to diagnose ileocecal, right side and transverse colon involvement in $100 \%, 70 \%$ and $50 \%$ of patients respectively while bowel US documented terminal ileum, ascending and transverse colon involvement in $100 \%$, $82 \%$ and $96 \%$ respectivelyin addition to free peritoneal fluid in $30 \%$ of cases.

All IBD subgroups $(\mathrm{n}=30)$ had BWT $>4 \mathrm{~mm}$; a value significantly higher than the control group; the latter having a mean $\leq 2 \mathrm{~mm}$. Patients with inactive CD exhibited a significant decrease in BWT; $4.85 \pm 0.72$; compared to those with active disease; $7.28 \pm 0.85 ; p$ value $<$ 0.01. This decrease in BWT was not observed in UC patients with inactive disease, $p$ value 0.18 as shown in (Figure 1). The mere presence of color signals in the affected bowel loop without specific quantification which were detected by Color Doppler study in $40 \%$ of CD patients; was the hallmark for activity.

Neither disease activity indices nor laboratory inflammatory markers was correlated with sonographic parameters of inflammation.

At initial assessment, color Doppler revealed an increase in the mean values for the diameter, PSV, EDV and blood volume of both the IMA and SMA among patients with pan-colitis and CD compared to the control groups; while RI of both mesenteric arteries and PI of the SMA were significantly decreased as shown in (Figure 2). Characteristically, the abnormal vascular parameters in IMA were related to both pan and left-sided colitis in contrast to those related to the SMA which was linked to the pan-colitis group only. On the other hand, none of the PV hemodynamic changes demonstrated a statistical significance in all IBD groups when compared to the control groups.

Doppler study was repeated in 10 IBD patients who achieved complete disease quiescence within six months duration. Hemodynamic changes in PV and both mesenteric arteries demonstrated a significant decrease in mean values for PSV, EDV and blood volume in contrast to those of RI and PI which exhibited a significant increase in their mean values compared to their original values during disease activity.

Receiver operator characteristic (ROC) curves were applied to assess the specificity and sensitivity of all vascular parameters and determine the best cutoff values that can help in the diagnosis of IBD (Figures 3 and $\mathbf{4}$, and Table 3).

\section{DISCUSSION}

In the gastroenterological setting, transabdominal bowel real time and Doppler US have been employed in the diagnosis, follow-up of IBD [13] and the assessment of postoperative recurrence of $\mathrm{CD}$ [14]. The diagnosis of $\mathrm{CD}$ and UC can be achieved with sensitivity and specificity of $75 \%-94 \%$ and $67 \%-100 \%$ respectively [15] in addition to the assessment of disease activity both in UC [16] and CD [17].

The present study was able to express the potential role of transabdominal bowel ultrasonography in evaluating the disease location, extent, activity and follow up of IBD patients. At initial presentation, the extent of IBD lesions was achieved as bowel US was able to diagnose left-sided and pan-colitis by $70 \%-85 \%$ accuracy whereas in the $\mathrm{CD}$, the diagnosis of terminal ileal lesions reached $100 \%$ with relatively lower accuracy; $82 \%-89.5 \%$, in the different colonic segments. This emphasizes the utility of bowel US in patients with CD rather than in UC due the frequent pelvic location of the latter. As regards the activity of IBD, bowel wall was significantly thickened in all IBD subgroups. Moreover, the presence of hypervascularization; as evident by an increase in splanchnic blood flow, increase in systolic and diastolic velocities,

Table 1. Laboratory parameters in all studied groups.

\begin{tabular}{|c|c|c|c|c|}
\hline Laboratory parameters & $\mathrm{UC}(\mathrm{n}=20)$ & $\mathrm{CD}(\mathrm{n}=10)$ & non IBD $(n=10)$ & Control $(n=10)$ \\
\hline Pus cells in stool $\left(/ \mathrm{cm}^{3}\right)$ & $38 \pm 6^{\mathrm{a}}$ & $33 \pm 5^{\mathrm{b}}$ & $5 \pm 3^{\mathrm{c}}$ & $0^{\mathrm{d}}$ \\
\hline $\mathrm{RBCs}$ in stool $\left(/ \mathrm{cm}^{3}\right)$ & $35 \pm 13^{\mathrm{a}}$ & $15 \pm 6^{\mathrm{b}}$ & $10 \pm 6^{\mathrm{b}}$ & $0^{\mathrm{c}}$ \\
\hline Hemoglobin $(\mathrm{g} / \mathrm{dl})$ & $11 \pm 1^{\mathrm{a}}$ & $12 \pm 1^{b}$ & $13 \pm 1^{\mathrm{c}}$ & $15 \pm 1^{\mathrm{d}}$ \\
\hline Albumin $(\mathrm{g} / \mathrm{dl})$ & $3.2 \pm 0.1^{\mathrm{a}}$ & $3.2 \pm 0.1^{\mathrm{a}}$ & $3.5 \pm 0.1^{\mathrm{b}}$ & $4.2 \pm 0.1^{\mathrm{c}}$ \\
\hline $\operatorname{ESR}(\mathrm{mm} / \mathrm{hr})$ & $39.2 \pm 7^{\mathrm{a}}$ & $41 \pm 4.4^{\mathrm{a}}$ & $19 \pm 1.4^{\mathrm{b}}$ & $5.2 \pm 3^{\mathrm{c}}$ \\
\hline
\end{tabular}

Values are expressed in mean $\pm \mathrm{SD}$. The mean difference is significant at the 0.05 level between each two different letters in each row. Similar letters indicate no significant difference. 
Table 2. Distribution of colonic lesions as detected by ultrasound compared to endoscopy in IBD patients $(n=20)$.

\begin{tabular}{ccc}
\hline Site of IBD lesions & Colonoscopy findings & US findings \\
\hline UC $(\mathrm{n}=20)$ & & \\
Left colitis & 10 & 7 \\
Pan-colitis & 10 & 4 \\
CD $(\mathrm{n}=10)$ & & \\
Terminal ileum & 10 & 10 \\
Caecum & 9 & 7 \\
Ascening colon & 7 & 4 \\
Transverse colon & 5 & 3 \\
\hline
\end{tabular}

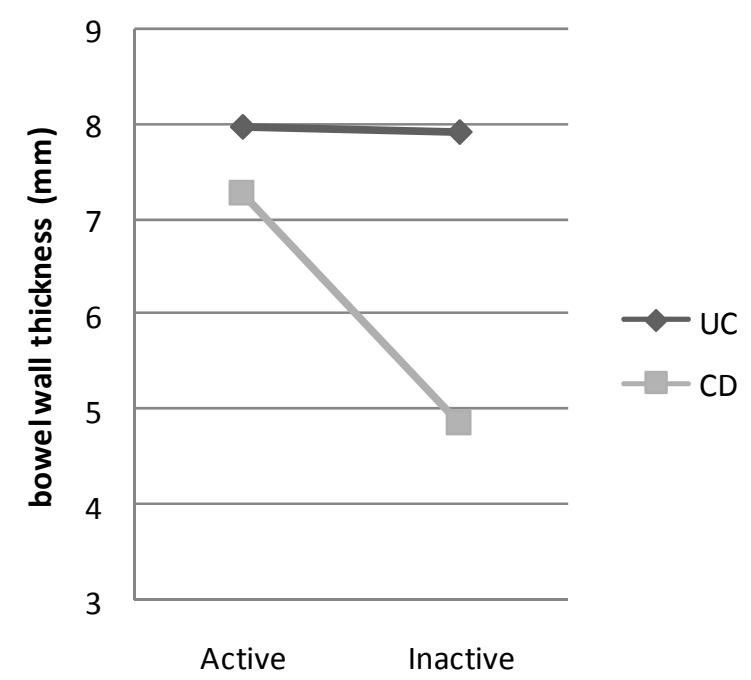

Figure 1. Bowel wall thickness in active and quiescent inflammatory bowel disease.

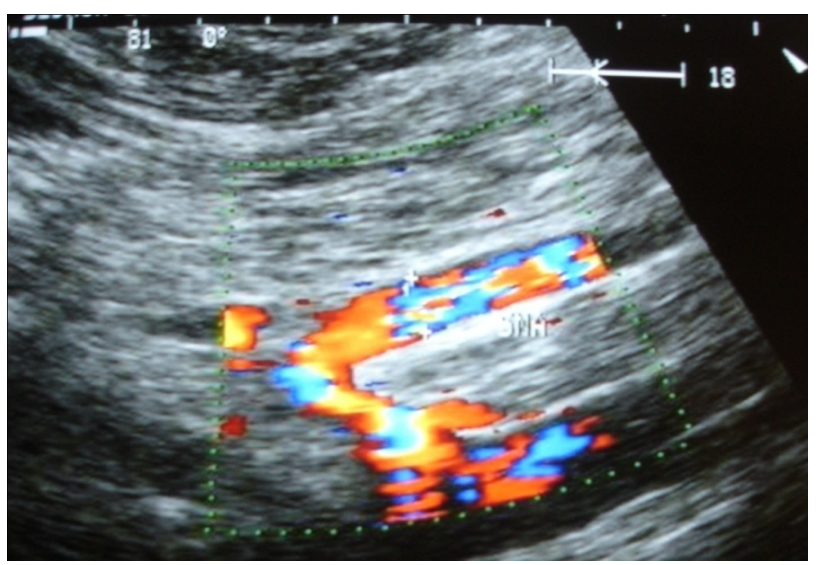

Figure 2. Color Doppler imaging demonstrating increased SMA diameter in active pan ulcerative colitis.

decrease in RI and PI in the mesenteric arteries; was the hallmark in the assessment of IBD activity. After complete disease remission, CD patients exhibited a significant decrease in BWT due to its trans-mural nature. Meanwhile, significant hemodynamic changes were found in all IBD patients in PV and both mesenteric arteries after disease quiescence, a finding consistent with de-

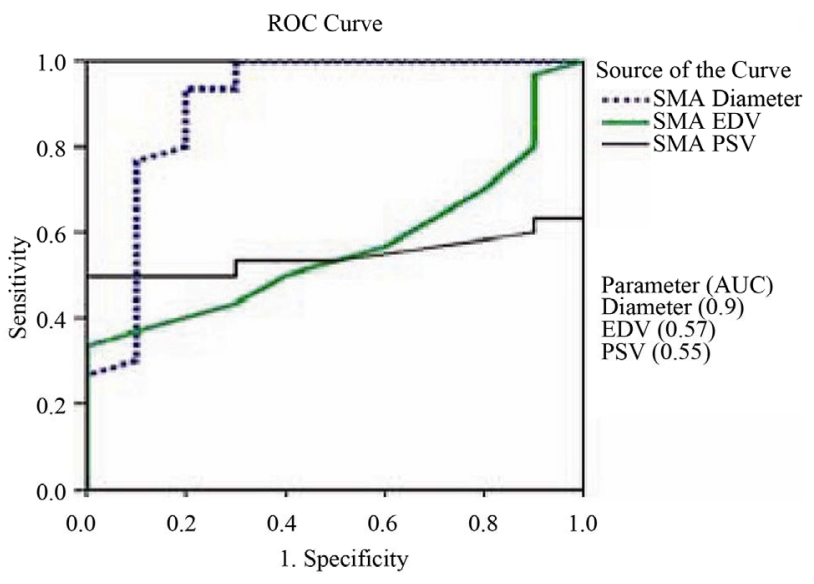

Figure 3. ROC curve of the different superior mesenteric artery vascular parameters (diameter, EDV and PSV) for differentiating between IBD patients and control groups.

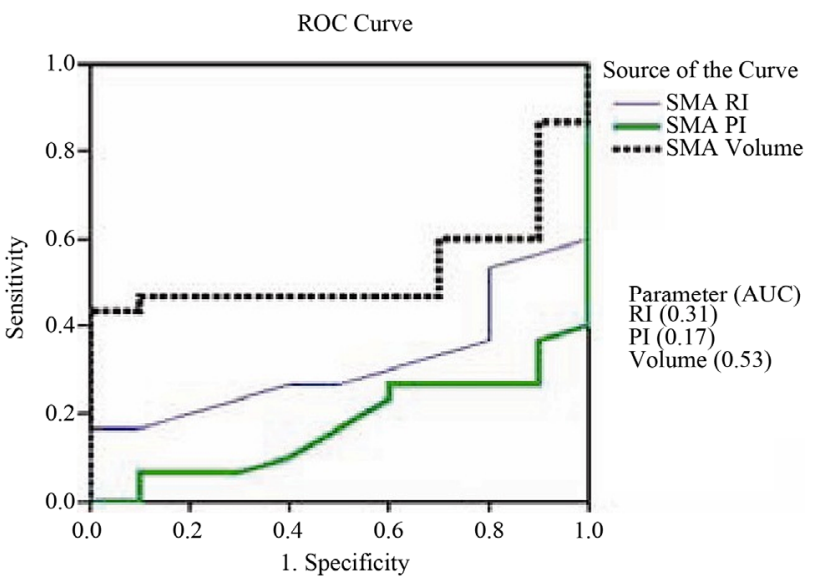

Figure 4. ROC curve of the different superior mesenteric artery vascular parameters (volume, PI and RI) for differentiating between IBD patients and control groups.

crease blood flow.

Disease location and extent could be variable between different IBD subgroups and even between different bowel segments in $\mathrm{CD}$. The relative lower accuracy in UC diagnosis could be due to the common rectal involvement which is relatively inaccessible by US. The variability in disease detection between the different bowel segments in $\mathrm{CD}$ was explained by the insufficient bowel distension and subsequent assessment of wall thickening; with best performance in the terminal ileum, lesser sensitivities in the ascending and transverse colon; $92 \%-97 \%$ with the least sensitivity; $33 \%-88 \%$; in the jejunum and proximal small bowel segments $[18,19]$. Prior studies were able to report an overall $84 \%-90 \%$ sensitivity $[20,21]$ and $78 \%-93 \%$ specificity for CD diagnosis, in addition to the strong correlation with the endoscopic and histopathological findings. Other studies revealed a wide range of sensitivity $(67 \%-96 \%)$ and specificity $(79 \%-100 \%)$ $[22,23]$. These differences were related to the variation in 
Table 3. Best cutoff values, sensitivity and specificity values for superior and inferior mesenteric artery vascular parameters.

\begin{tabular}{cccccc}
\hline Vascular parameter & Best cutoff & Sensitivity & Specificity & AUC & $p$ value \\
\hline SMA diameter $(\mathrm{mm})$ & 6 & $80 \%$ & $80 \%$ & 0.9 & 0.002 \\
IMA diameter $(\mathrm{mm})$ & 3.35 & $93 \%$ & $100 \%$ & 0.98 & $<0.01$ \\
IMA EDV $(\mathrm{m} / \mathrm{sec})$ & 1.12 & $97 \%$ & $100 \%$ & 0.99 & $<0.01$ \\
IMA PSV $(\mathrm{m} / \mathrm{sec})$ & 1.276 & $83 \%$ & $100 \%$ & 0.93 & $<0.01$ \\
\hline
\end{tabular}

SMA: superior mesenteric artery; IMA: inferior superior mesenteric artery; PSV: peak systolic velocity; EDV: end diastolic velocity; RI: resistivity index; PI: pulsatility index; AUC: area under the curve; $p$ value was considered significant when $\leq 0.05$.

the study design, population characteristics, reference standards and lack of a control population which makes it difficult to achieve a comprehensive evaluation of sensitivity and specificity of the US.

BWT, a characteristic feature of IBD, is theoretically expected to increase with the trans-mural inflammation in $C D$ rather than the exclusive superficial mucosal inflammation in UC. However, BWT is considered to be a characteristic feature of $\mathrm{UC}$ as well. In $\mathrm{CD}$, US may show BWT $\geq 20 \mathrm{~mm}$ with possible loss of stratification [13]; whereas the mural stratification is preserved in most UC patients. This feature can differentiate between $\mathrm{CD}$ and UC, although it was regarded to be non-sufficient for differentiation in most of the studies [24]. In accordance with our results, BWT detected by bowel US was correlated with the clinical and endoscopic activity of UC and could yield high sensitivity in detecting macroscopic lesions when compared to endoscopic and histological findings. BWT significantly decreased (7.3 \pm $1.9 \mathrm{~mm}$ versus $5.1 \pm 1.2 \mathrm{~mm} ; \mathrm{p}<0.001)$ in patients who experienced clinical improvement after treatment, but this decrease was not evident in those showing no significant clinical improvement. In $\mathrm{CD}$, bowel loop wall was significantly thicker than the control group; $4.9 \pm 2.7$ vs. $<2.0 \mathrm{~mm}$; with greater values in active compared to the inactive phases; $5.8 \pm 2.9$ vs. $4.3 \pm 2.2 \mathrm{~mm}$ respectively; $p<0.001[25,26]$. Similarly, significant differences in BWT between different phases of IBD were reported by several authors [27-29] and were explained by the presence of edema, muscular spasm, and submucosal cellular inflammation inactive phases in the subsequent development of fibrosis in inactive phases [30]. Moreover, the different cutoff values for BWT revealed a decrease in sensitivity from $88 \%$ to $75 \%$ with a slight increase in specificity from 93\% - 97\% when a cutoff value of $4 \mathrm{~mm}$ was used instead of $3 \mathrm{~mm}$ [31] and both sensitivity and specificity were improved with the use of the 4-mm compared to 5-mm threshold [32].

Our results illustrated the presence of hypervascularization and the increase in mesenteric blood flow which was related to the extent of colonic involvement, IBD activity and the favorable response to treatment. Thus, Doppler sonography was able to demonstrate splanchnic hemodynamic changes in active IBD patients and can differentiate between active and quiescent cases. In agreement with our results, other authors correlated hemodynamic changes to disease activity, both in UC [16] and CD [17] as demonstrated by Doppler sonography which was useful in IBD diagnosis even on normal colonoscopy findings [33]. Increase PV blood flow and gut vascularity and decrease SMA RI were able to differentiate between active and quiescent UC [19] and were demonstrated in active CD [34]. All hemodynamic changes in SMA were related to pan-colitis rather than to leftsided colitis; SMA blood flow is likely to be fairly normal when the inflammation is limited to the left colon or rectum [16,35]. Similarly, an increase in mesenteric arteries velocity with the subsequent decrease towards the normal values was expressed in IBD patients during remission [36], in UC [37] and in determining CD activity [38]. CD activity was related to the intensity of color Doppler signals [28], decrease RI in mural arteries and increase PV blood flow [39]. Moreover, active CD was predicted by $86 \%$ at a cut off value for RI and PI at $<0.79,<1.56$ respectively [40] and relapse was represented by an absence of PI augmentation even when clinical basis indicates remission phase. The present study was able to elicit the cutoff values for SMA diameter (6 $\mathrm{mm})$, IMA diameter (3.35 mm), EDV, PSV and blood flow volume that were able to diagnose cases with IBD with high sensitivity and specificity.

In the current study, supplementary features in the form of free fluid and the presence of color signals in the affected bowel loops were helpful in the diagnosis of IBD specially among CD patients. This was previously noted by other authors who appreciated few additional features such as mesenteric hypertrophy, free fluid or lymph nodes that could substantially improve the performance of the US in the diagnosis of CD [41].

In variance to the present study, Doppler analysis was not correlated with clinical activity as demonstrated by Byrne et al. who could not demonstrate any correlation between SMA RI with either endoscopic or clinical disease severity [38]. This may be attributed to the different spectra of disease severity between the study cohorts, or to methodological flaw. In routine clinical practice, Doppler measurements in the SMA are not always reliable due to influence of age, presence of atherosclerosis, and 
the length of the affected segment [38]. Moreover, the determination of blood flow in CD is technically demanding, is not feasible in all patients and is time consuming. This raises more questions to the possibility of the routine application of this test in clinical practice [42] which was further supported when considering the insignificant hemodynamic changes related to the PV diameter, velocity and blood flow volume in all our IBD subgroups. However, a significant decrease in all PV vascular parameters was achieved on disease remission, as previously demonstrated in other studies $[3,43]$.

The current study expresses some limitations, the small number of IBD patients either in activity or remission, the lack of accurate endoscopic grading scoring systems, the lack of information about the disease severity and its relation to the maximal BWT. Furthermore, the use of convex and linear probes of higher frequency US might have added more detailed information about the pathology of bowel loops involved in addition to their content, peristalsis, and compressibility. Moreover, the assessment of the IMA was frequently concealed by bowel gases and necessitated re-evaluation and transabdominal bowel ultrasonography can miss clinically relevant pathological changes in patients with IBD mostly due to difficulty in assessing certain anatomical regions.

In conclusion, though establishing IBD diagnosis should be based on endoscopic and histological findings, this preliminary study had highlighted the possible application of transabdominal bowel ultrasonography as a valuable; well tolerated method to assist in the diagnosis, location, determination of disease activity and follow-up of IBD.

\section{REFERENCES}

[1] Vucelic, B. (2009) Inflammatory bowel diseases: Controversies in the use of diagnostic procedures. Digestive Diseases and Sciences, 27, 269-277. doi:10.1159/000228560

[2] MacKalski, B.A. and Bernstein, C.N. (2006) New diagnostic imaging tools for inflammatory bowel disease. Gut, 55, 733-741. doi:10.1136/gut.2005.076612

[3] Bass, N., Smith, L.H. and Van Dyke, R.W. (1993) Inflammatory bowel disease. In: Andreoli, T.E., Bennet, J.C., Carpenter, C.C.J., Plum, F. and Smith, L.H., Eds., Cecil Essentials of Medicine, WB Saunders, Philadelphia, 299.

[4] Marshall, J.B. and Barthel, J.S. (1993) The frequency of total colonoscopy and terminal ileal intubation in the 1990s. Gastrointestinal Endoscopy, 39, 518-520. doi:10.1016/S0016-5107(93)70162-5

[5] Ambrosini, R., Barchiesi, A., Di Mizio, V., et al. (2007) Inflammatory chronic disease of the colon: How to image. European Journal of Radiology, 61, 442-448. doi:10.1016/j.ejrad.2006.07.028

[6] Nylund, K., Hausken, T. and Gilja, O.-H. (2010) Ultra- sound and inflammatory bowel disease. Ultrasound Quarterly, 26, 3-15. doi:10.1097/RUQ.0b013e3181ce0929

[7] Di Sabatino, A., Armellini, E. and Corazza, G.R. (2004) Doppler sonography in the diagnosis of inflammatory bowel disease. Digestive Diseases and Sciences, 22, 6366. doi: $10.1159 / 000078736$

[8] Schroeder, K.W., Tremaine, W.J. and Ilstrup, D.M. (1987) Coated oral 5-aminosalcylic acid therapy for mildly to moderately active ulcerative colitis. New England Journal of Medicine, 317, 1625-1629. doi:10.1056/NEJM198712243172603

[9] Best, W.R., Becktel, J.M., Singleton, J.W., et al. (1976) Development of a Crohn's disease activity index. National Cooperative Crohn's Disease Study. Gastroenterology, 70, 439-444.

[10] Sandborn, W.J., Feagan, B.G., Hanauer, S.B., et al. (2002) A review of activity indices and efficacy endpoints for clinical trials of medical therapy in adults with Crohn's disease. Gastroenterolgy, 122, 512-530. doi:10.1053/gast.2002.31072

[11] Jeffrey, R.B., Jain, K.A. and Nghiem, H.V. (1994) Sonographic diagnosis of acute appendicitis: Interpretative pitfalls. American Journal of Roentgenology, 162, 55-59. doi:10.2214/ajr.162.1.8273690

[12] Dietrich, C.F., Barreiros, A.P., Nuernberg, D., et al. (2008) Perianal ultrasound (in German). Zeitschrift Fur Gastroenterologie, 46, 625-630. doi:10.1055/s-2008-1027518

[13] Cammarota, T., Sarno, A., Robotti, D., et al. (2009) US evaluation of patients affected by IBD: How to do it, methods and findings. European Journal of Radiology, 69, 429-437. doi:10.1016/j.ejrad.2008.11.008

[14] Maconi, G., Radice, E., Greco, S., et al. (2006) Bowel ultrasound in Crohn's disease. Best Practice \& Research Clinical Gastroenterology, 20, 93-112. doi:10.1016/j.bpg.2005.09.001

[15] Lim, J.H., Ko, Y.T., Lee, D.H., et al. (1994) Sonography of inflammatory bowel disease: Findings and value in differential diagnosis. American Journal of Roentgenology, 163, 343-347. doi:10.2214/ajr.163.2.8037027

[16] Siğirci, A., Baysal, T., Kutlu, R., et al. (2001) Doppler sonography of the inferior and superior mesenteric arteries in ulcerative colitis. Journal of Clinical Ultrasound, 29, 130-139.

doi:10.1002/1097-0096(200103/04)29:3<130::AID-JCU1 012>3.0.CO;2-X

[17] Spalinger, J., Patriquin, H., Miron, M.C., et al. (2000) Doppler US in patients with Crohn disease: Vessel density in the diseased bowel reflects disease activity. Radiology, 217, 787-791.

[18] Bozkurt, T., Richter, F. and Lux, G. (1994) Ultrasonography as a primary diagnostic tool in patients with inflammatory disease and tumors of the small intestine and large bowel. Journal of Clinical Ultrasound, 22, 85-91. doi:10.1002/jcu.1870220204

[19] Maconi, G., Parente, F., Bollani, S., et al. (2006) Abdominal ultrasound in the assessment of extent and activity of Crohn's disease: Clinical significance and implica- 
tion of bowel wall thickening. American Journal of Gastroenterology, 91, 1604-1609.

[20] Astegiano, M., Bresso, F., Cammarota, T., et al. (2001) Abdominal pain and bowel dysfunction: Diagnostic role of intestinal ultrasound. European Journal of Gastroenterology \& Hepatology, 13, 927-931. doi:10.1097/00042737-200108000-00009

[21] Parente, F., Greco, S., Molteni, M., et al. (2003) Bowel ultrasound as primary imaging procedure in patients with suspected inflammatory bowel disorders. Alimentary Pharmacology \& Therapeutics, 18, 1009-1016. doi:10.1046/j.1365-2036.2003.01796.x

[22] Hollerbach, S., Geissler, A., Schiegl, H., et al. (1998) The accuracy of abdominal ultrasound in the assessment of bowel disorders. Scandinavian Journal of Gastroenterology, 33, 1201-1208. doi:10.1080/00365529850172575

[23] Rispo, A., Imbriaco, M., Celentano, L., et al. (2005) Noninvasive diagnosis of small bowel Crohn's disease: Combined use of bowel sonography and Tc-99m-HMPAO leukocyte scintigraphy. Inflammatory Bowel Diseases, 11, 376-382. doi:10.1097/01.MIB.0000164020.65106.84

[24] Limberg, B. and Osswald, B. (1994) Diagnosis and differential diagnosis of ulcerative colitis and Crohns disease by hydrocolonicsonography. American Journal of Gastroenterology, 89, 1051-1057.

[25] Maconi, G., Ardizzone, S., Parente, F., et al. (1999) Ultrasonography in the evaluation of extension, activity, and follow-up of ulcerative colitis. Scandinavian Journal of Gastroenterology, 34, 1103-1107. doi:10.1080/003655299750024904

[26] Heyne, R., Rickes, S., Bock, P., et al. (2002) Non-invasive evaluation of activity in inflammatory bowel disease by power Doppler sonography. Zeitschrift Fur Gastroenterologie, 40, 171-175. doi:10.1055/s-2002-22325

[27] Sjekavica, I., Barbarić-Babić, V., Krznarić, Z., et al. (2007) Assessment of Crohn's disease activity by doppler ultrasound of superior mesenteric artery and mural arteries in thickened bowel wall: Cross-sectional study. Croatian Medical Journal, 48, 822-830. doi:10.3325/cmj.2007.6.822

[28] Martínez, M.J., Ripollés, T., Paredes, J.M., et al. (2009) Assessment of the extension and the inflammatory activity in Crohn's disease: Comparison of ultrasound and MRI. Abdominal Imaging, 34, 141-148. doi:10.1007/s00261-008-9365-y

[29] Haber, H.P., Busch, A., Ziebach, R., et al. (2000) Bowel wall thickness measured by ultrasound as a marker of Crohn's disease activity in children. Lancet, 355, 12391240. doi:10.1016/S0140-6736(00)02092-4

[30] Fraquelli, M., Colli, A., Casazza, G., et al. (2005) Role of US in detection of Crohn disease: Meta-analysis. Radiology, 236, 95-101. doi:10.1148/radiol.2361040799

[31] Horsthuis, K., Bipat, S., Bennink, R.J., et al. (2008) Inflammatory bowel disease diagnosed with US, MR, scintigraphy, and CT: Meta-analysis of prospective studies. Radiology, 247, 64-79. doi:10.1148/radiol.2471070611

[32] Trattenero, C., Losco, A., Fraquelli, M., et al. (2009)
Small bowel ultrasound and computed tomography enterography: Characteristics of bowel wall and mesenteric tissue in the first diagnosis of ileal Crohn's disease. Gastroenterology, 136, Abstract 651.

[33] Lukic-Kostic, L., Jovic, J. and Sekulovic, S. (2006) Significance of ultrasonography of the terminal ileum in moderate Crohn's disease. Vojnosanitetski Pregled, 63, 787-792. doi:10.2298/VSP0609787L

[34] Epifanio, M., Baldisserotto, M., Spolidoro, J.V., et al. (2008) Grey-scale and colour Doppler sonography in the evaluation of children with suspected bowel inflammation: Correlation with colonoscopy and histological findings. Clinical Radiology, 63, 968-978. doi:10.1016/j.crad.2008.02.009

[35] Giovagnorio, F., Diacinti, D. and Vernia, P. (1998) Doppler sonography of the superior mesenteric artery in Crohn's disease. American Journal of Roentgenology, 170, 123-126. doi:10.2214/ajr.170.1.9423614

[36] Mirk, P., Palazzoni, G. and Giamondo, P. (1999) Doppler sonography of hemodynamic changes of the inferior mesenteric artery in inflammatory bowel disease: Preliminary data. American Journal of Roentgenology, 173, 381387.

[37] Sigirci, A., Baysal, T., Kutlu, R., et al. (2003) Doppler sonography of the inferior and superior mesenteric arteries in ulcerative colitis. Journal of Clinical Ultrasound, 29, 130-139. doi:10.1002/1097-0096(200103/04)29:3<130::AID-JCU1 012>3.0.CO;2-X

[38] Byrne, M.F., Farrell, M.A., Abass, S., et al. (2001) Assessment of Crohn's disease activity by Doppler sonography of the superior mesenteric artery, clinical evaluation and the Crohn's disease activity index: A prospective study. Clinical Radiology, 56, 973-978. doi:10.1053/crad.2001.0794

[39] Yekeler, E., Danalioglu, A., Movasseghi, B., et al. (2005) Crohn disease activity evaluated by Doppler ultrasonography of the superior mesenteric artery and the affected small-bowel segments. Journal of Ultrasound in Medicine, 24, 59-65.

[40] Karouia, S., Nouirab, K., Serghinia, M., et al. (2010) Assessment of activity of Crohn's disease by Doppler sonography of superior mesenteric artery flow. Journal of Crohn's and Colitis, 4, 334-340. doi:10.1016/j.crohns.2009.12.011

[41] Maccioni, F., Colaiacomo, M.C. and Parlanti, S. (2005) Ulcerative colitis: Value of MR imaging. Abdominal Imaging, 30, 584-592. doi:10.1007/s00261-004-0284-2

[42] Parente, F., Greco, S., Molteni, M., et al. (2005) Imaging inflammatory bowel disease using bowel ultrasound. European Journal of Gastroenterology \& Hepatology, 17, 283-291.

[43] Bolondi, L., Gaiani, S., Brignola, C., et al. (1992) Changes in splanchnic hemodynamics in inflammatory bowel disease. Non-invasive assessment by Doppler ultrasound flowmetry. Scandinavian Journal of Gastroenterology, 27, 501-507. doi:10.3109/00365529209000112 


\section{ABBREVIATIONS}

IBD: Inflammatory Bowel Disease

UC: Ulcerative Colitis

CD: Crohn's Disease

IC: Indeterminate Colitis

US: Ultrasonography

CRP: C-Reactive Protein

ESR: Erythrocyte Sedimentation Rate

CDAI: Crohn's Disease Activity Index
BWT: Bowel Wall Thickness

PV: Portal Vein

SMA: Superior Mesenteric Artery

IMA: Inferior Mesenteric Artery

PSV: Peak Systolic Velocity

EDV: End Diastolic Velocity

RI: Resistivity Index

PI: Pulsatility Index

ROC: Receiver Operator Characteristic 